\title{
Thermally induced suppression of interchain interactions in dilute aqueous solutions of conjugated polyelectrolyte rotaxanes and their analogues
}

Giulia Tregnago, Ali Afshar, Shane O. McDonnell, Harry L. Anderson, and Franco Cacialli

Citation: Appl. Phys. Lett. 111, 083301 (2017); doi: 10.1063/1.4993457

View online: http://dx.doi.org/10.1063/1.4993457

View Table of Contents: http://aip.scitation.org/toc/apl/111/8

Published by the American Institute of Physics

\section{Articles you may be interested in}

High-resolution charge carrier mobility mapping of heterogeneous organic semiconductors

Applied Physics Letters 111, 083302 (2017); 10.1063/1.4999762

Purcell-enhancement of the radiative PL decay in perylenediimides by coupling with silver nanoparticles into waveguide modes

Applied Physics Letters 111, 081102 (2017); 10.1063/1.4999325

Probing material conductivity in two-terminal devices by resistance difference

Applied Physics Letters 111, 083501 (2017); 10.1063/1.5000150

Persistent luminescence nanothermometers

Applied Physics Letters 111, 081901 (2017); 10.1063/1.4990873

Fast optical work-function tuning at an organic/metal interface

Applied Physics Letters 111, 081601 (2017); 10.1063/1.4999436

Electric field control of ferromagnetism at room temperature in $\mathrm{GaCrN}(\mathrm{p}-\mathrm{i}-\mathrm{n})$ device structures

Applied Physics Letters 111, 082402 (2017); 10.1063/1.4986431

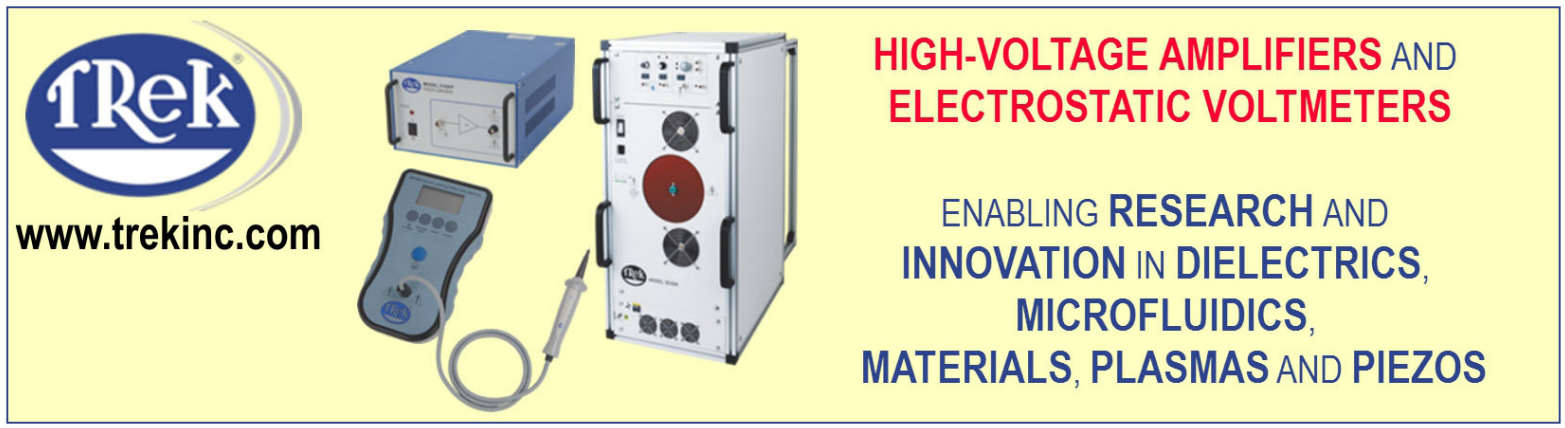




\title{
Thermally induced suppression of interchain interactions in dilute aqueous solutions of conjugated polyelectrolyte rotaxanes and their analogues
}

\author{
Giulia Tregnago, ${ }^{1}$ Ali Afshar, ${ }^{1,2}$ Shane O. McDonnell, ${ }^{3}$ Harry L. Anderson, ${ }^{3}$ \\ and Franco Cacialli ${ }^{1, a)}$ \\ ${ }^{1}$ Department of Physics and Astronomy and London Centre for Nanotechnology, University College London, \\ London WC1E 6BT, United Kingdom \\ ${ }^{2}$ Department of Chemistry, Imperial College London, London SW7 2AZ, United Kingdom \\ ${ }^{3}$ Department of Chemistry, University of Oxford, Oxford OXI 3TA, United Kingdom
}

(Received 28 June 2017; accepted 2 August 2017; published online 22 August 2017)

\begin{abstract}
We use steady-state and nanosecond time-resolved photoluminescence spectroscopy to investigate the evolution of packing interactions in dilute solutions of a sulfonated poly(diphenylenevinylene) lithium salt and its cyclodextrin-threaded polyrotaxanes as a function of the threading ratio (TR) when increasing the temperature from 10 to $40^{\circ} \mathrm{C}$. Contrary to the expectation of a temperatureinduced increase of packing and aggregation, supported by previous Raman studies identifying a temperature-induced reduction in the inter-phenyl torsion angles, we find clear spectral (photoluminescence blue-shift and narrowing) and dynamic (shorter lifetimes and reduced weight of the longlived components) signatures of a reduction of interchain interactions for the polyelectrolytes at higher temperatures with TR up to 1.3. Published by AIP Publishing.

[http://dx.doi.org/10.1063/1.4993457]
\end{abstract}

Luminescent conjugated polyelectrolytes (CPEs) constitute an intriguing class of conjugated semiconductors with significant potential for a variety of applications spanning from biosensing to organic electronics and photonics. ${ }^{1-3}$ Crucial to fulfilling their potential is however control of their optical properties, which in turn requires accurate knowledge of the dependence of these properties on the details of the intramolecular (covalent) connectivity and side-chain functionalization, and of the inter- and supra-molecular organisation. ${ }^{4}$ The formation of weakly emissive aggregates and/or weakly bound excimers leads to a reduction of the photoluminescence quantum yield (PLQY), accompanied by a redshift and linewidth broadening of the emission spectrum, which may affect the performance and colour purity of lightemitting diodes (LEDs) incorporating these materials. In addition to these spectroscopic changes, weakly emissive aggregates and excimers/weakly bound charge-transfer states are also characterised by longer lifetimes, from a few ns to over 10-20 ns depending on the extent of the interaction. Conversely, such a combination of spectroscopic and dynamic photoluminescence (PL) features has been taken as a powerful indicator of the presence/suppression of interchain interactions in much recent literature. ${ }^{5-8}$ Various strategies have been demonstrated to suppress the formation of interchain states, thus enhancing the photoluminescence efficiency and blue-shifting the emission: from side-chain engineering, ${ }^{9,10}$ to encapsulation of the active polymer chains into "inert" macrocycles (e.g., cyclodextrins), ${ }^{11,12}$ to use of co-solvent water/alcohol systems. ${ }^{13,14}$

Interestingly, variations in temperature can sensitively trigger changes in the intra- and inter-chain interactions between the conjugated backbones that can be probed by monitoring their impact on the optical properties, making

${ }^{\text {a)} E-m a i l: ~ f . c a c i a l l i @ u c l . a c . u k ~}$ these properties very responsive to heat stimuli for a variety of conjugated polymers. ${ }^{15-19}$ Previously, Raman and resonant Raman spectroscopies have been used to study thermochromism of sulfonated diphenylenevinylene derivatives, and it was concluded that these polymers undergo a conformational change into a more planar geometry when the temperature is increased to $40{ }^{\circ} \mathrm{C}$, apparently favouring intermolecular interactions. ${ }^{20}$ However, the occurrence of such interactions and related states must be confirmed by analyses of the thermally induced alterations to spectra and excitedstate dynamics.

To this end, we investigated the optical properties of sulfonated poly(diphenylene-vinylene) lithium salt [PDV.Li, see Fig. 1(a)] and cyclodextrin polyrotaxane analogues (PDV.Li $\subset \beta$-CD, with different threading ratios, $\mathrm{TR}=0.5$, $1.3,1.8,2.0)$ when the increase in temperature from 10 to $40{ }^{\circ} \mathrm{C}$. We chose to work with solutions at a concentration of $10^{-4} \mathrm{~g}^{-1}$ concentration, which is dilute enough to minimise, albeit not eliminate, interchain interactions across the whole range of TRs. We found that the PL and absorption spectra are blue-shifted, and that there is a clear reduction of the weight of the long-lived component of the PL decay upon heating for the polyelectrolytes with TR up to 1.3 , thereby pointing to a clear reduction of interchain interactions even for such mild temperature variations. The most threaded rotaxanes $(\mathrm{TR}=2)$ are virtually insensitive to temperature variations, confirming the effectiveness of this supramolecular approach for the minimization of the interchain interactions.

The synthesis of sulfonate substituted poly(diphenylenevinylene) lithium salt (PDV.Li) and its cyclodextrin polyrotaxane derivative (PDV.Li $\subset \beta$-CD) used in this work is described elsewhere. ${ }^{21}$ All data shown were taken for PDV.Li (also indicated with $\mathrm{TR}=0$ ) and PDV.Li $\subset \beta$-CD $(\mathrm{TR}=0.5,1.3,1.8$, and 2.0$)$ in buffer solutions $(11.6 \mathrm{mM}$ 
a

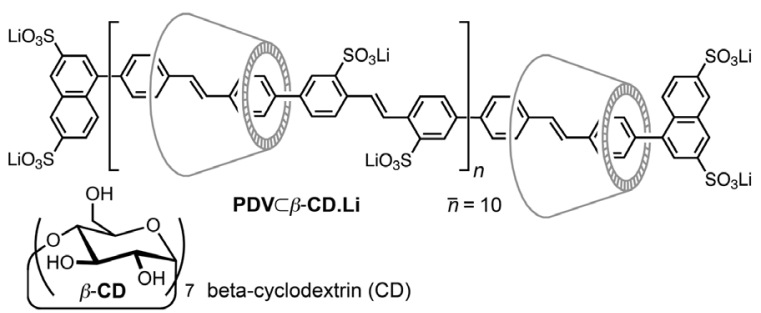

b

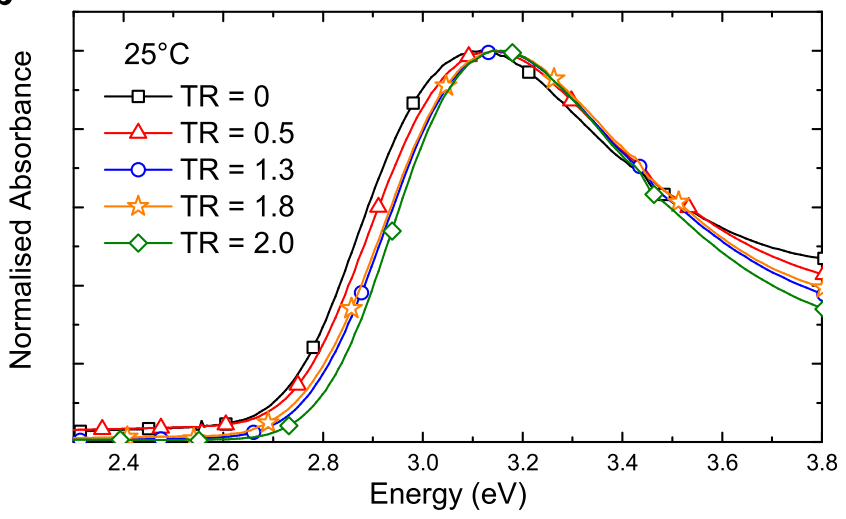

FIG. 1. Chemical structures of the sulfonate-substituted poly(diphenylenevinylene) lithium salt rotaxane (PDV.Li $\subset \beta$-CD) and cyclodextrin $(\beta$-CD) macrocycle. PDV.Li is the same as PDV.Lic $\subset$-CD except without the threaded cyclodextrin rings (a). Normalised absorption spectra of $10^{-2} \mathrm{~g}^{-1}$ solution of PDV.Li (TR $=0$, black squares) and PDV.LiC $\beta$-CD with different threading ratio, $\mathrm{TR}=0.5$ (red triangles), 1.3 (blue circles), 1.8 (orange stars), and 2.0 (green diamonds). The spectra were recorded at $25^{\circ} \mathrm{C}(\mathrm{b})$.

$\mathrm{NaOH} / 20 \mathrm{mM} \mathrm{KH}_{2} \mathrm{PO}_{4}$ ) at polymer concentration of $10^{-2} \mathrm{~g}^{-1}$. The threading ratio (TR) is defined as the cyclodextrin macrocycles per repeat unit. The buffer solution is used to keep the $\mathrm{pH}$ at 7 and to provide a constant ionic strength. Higher ionic strengths tend to enhance the hydrophobic effect, with increased salt concentration, thereby resulting in increased aggregation of non-polar organic molecules. An absorption coefficient of $\varepsilon=15\left(\mathrm{~g} \mathrm{l}^{-1}\right)^{-1} \mathrm{~mm}^{-1}$, or $9 \times 10^{5} \mathrm{M}^{-1} \mathrm{~cm}^{-1}$, at $404 \mathrm{~nm}$ was previously estimated measuring the absorption of PDV.Li solution at various polymer concentrations. ${ }^{20}$ Such a coefficient allows the determination of the concentration of the solutions investigated in this work by using UV-Vis absorption spectroscopy (Agilent 8543 UV-Visible spectrophotometer). To reduce interchain interactions between polymer chains with a view to studying conformational changes, the solutions were then further diluted to $10^{-4} \mathrm{~g} \mathrm{l}^{-1}$. At this concentration, the interchain species are nearly completely suppressed also in the case of the unthreaded polymer. ${ }^{22}$ The steady-state PL spectra were recorded after exciting at $405 \mathrm{~nm}$ with a diode laser by means of an ANDOR-Shamrock spectrograph coupled with an ANDOR-Newton charge-coupled device (CCD) unit. Decay dynamics were studied with a time-correlated singlephoton counting (TCSPC) spectrometer using a ps-pulsed diode laser at $371 \mathrm{~nm}$ (Edinburgh Instruments EPL-375) and a F-900 TCSPC unit (temporal resolution $\sim 150 \mathrm{ps)}$ ) with a photomultiplier tube coupled to a monochromator. The temperature-dependent study was conducted by using a resistive heater and a Peltier cooler and the temperature was constantly monitored with a Pt (Pt100) thermistor.
The room-temperature absorption spectra of a solution of $10^{-2} \mathrm{~g}^{-1}$ of PDV.Li and PDV.LiC $\beta$-CD with various threading ratios are reported in Fig. 1(b). As previously reported, ${ }^{23}$ the polyrotaxanes absorption is blue-shifted $\left(\mathrm{E}_{\mathrm{abs}, \mathrm{TR}=0}=3.07 \mathrm{eV}, \mathrm{E}_{\mathrm{abs}, \mathrm{TR}=2}=3.13 \mathrm{eV}\right)$ and slightly narrower compared to that of the unthreaded polymer $\left(\mathrm{FWHM}_{\mathrm{TR}=0}=0.57 \mathrm{eV}, \mathrm{FWHM}_{\mathrm{TR}=2}=0.52 \mathrm{eV}\right)$. A similar trend is also observable as a function of the threading ratio, as expected for a progressively more effective suppression of intermolecular interactions with TR. Besides the blue-shift we also note that rotaxination of the polymers does not affect the overall shape of the spectrum, thus confirming that the cyclodextrins are optically inert and preserve the spectroscopic characteristics of the isolated chain.

Turning now to the temperature-dependent data, in Figs. 2(a)-2(f), we report both the parameters extracted from the photoluminescence spectra of $10^{-4} \mathrm{~g}^{-1}$ solutions of the PDV.Li and related polyrotaxanes with various threading ratios [Figs. 2(a)-2(c)], and representative full spectra [Figs. 2(d)-2(f)] for TR $=0,0.5,2.0$, respectively. More specifically, Fig. 2(a) shows the energy of the $0-0$ transition and the change of its position upon temperature variation (inset), whereas in Fig. 2(b), we report the full-width at half maximum (FWHM) of the emission, and (c) the evolution of the $\mathrm{I}_{0-\mathrm{n}} / \mathrm{I}_{0-0}$ ratio (where the $0-\mathrm{n}$ is the most intense transition excluding the $0-0)$.

Both unthreaded and "poorly threaded" $(\mathrm{TR} \leq 1.3)$ polymers exhibit a marked blue shift, an increase of the $\mathrm{I}_{0-\mathrm{n}} / \mathrm{I}_{0-0}$ ratio and a decrease of the FWHM [Figs. 2(a)-2(c)] when the temperature increases from $10^{\circ} \mathrm{C}$ to $40^{\circ} \mathrm{C}$ that clearly point to a reduction of interchain species. ${ }^{24}$ We note that the thermochromic effect is stronger for these rotaxanes compared to those with higher TR $[\sim 1.1 \mathrm{meV} / \mathrm{K}$ for $\mathrm{TR} \leq 1.3$ and $\sim 0.6 \mathrm{meV} / \mathrm{K}$ for $\mathrm{TR} \geq 1.8$, respectively, see inset in Fig. 2(a)]. This is in agreement with previous findings where thermally induced modification of the dihedral torsion angles along the chains were evident in analogous polymers with low threading ratios. ${ }^{20}$ However, we cannot exclude that even though the 0-0-transition energy is least affected by packing and aggregation, the reduction of interchain interactions in unthreaded and "poorly threaded" polymers may also contribute to the blue-shift of the $0-0$ peak. The thermochromic effect of rotaxanes with TR $\geq 1.8$ is surprisingly similar to that of unsubstituted poly( $p$-phenylene vinylene) $(\sim 0.4-0.5 \mathrm{meV} / \mathrm{K}$ for PPV $){ }^{25}$ Even though PDV.Li $\subset \beta$-CD characterised by such threading ratios have been suggested to be resistant to conformational alterations with temperature, ${ }^{20}$ thermochromic effects in these systems are not unexpected and have been reported for other PPV derivatives. ${ }^{16,26}$

Blue-shifted and narrower PL spectra and a decreased $\mathrm{I}_{0-\mathrm{n}} / \mathrm{I}_{0-0}$ ratio are also observable upon progressive encapsulation of PDV.Li into cyclodextrin macrocycles due to a reduced tendency to aggregate of the conjugated backbones. ${ }^{7}$ Even if at a concentration of $10^{-4} \mathrm{~g} \mathrm{l}^{-1}$ the polymers are considered highly diluted, we can still detect signs of aggregation, albeit minimal, in line with previously reported data. ${ }^{22}$ The reduction of interchain interactions upon temperature increase is strongest for the unthreaded polymer and its rotaxinated counterpart with the lowest TR $(\mathrm{TR}=0.5)$, and the effect is comparable in nature to that obtained by forcing 

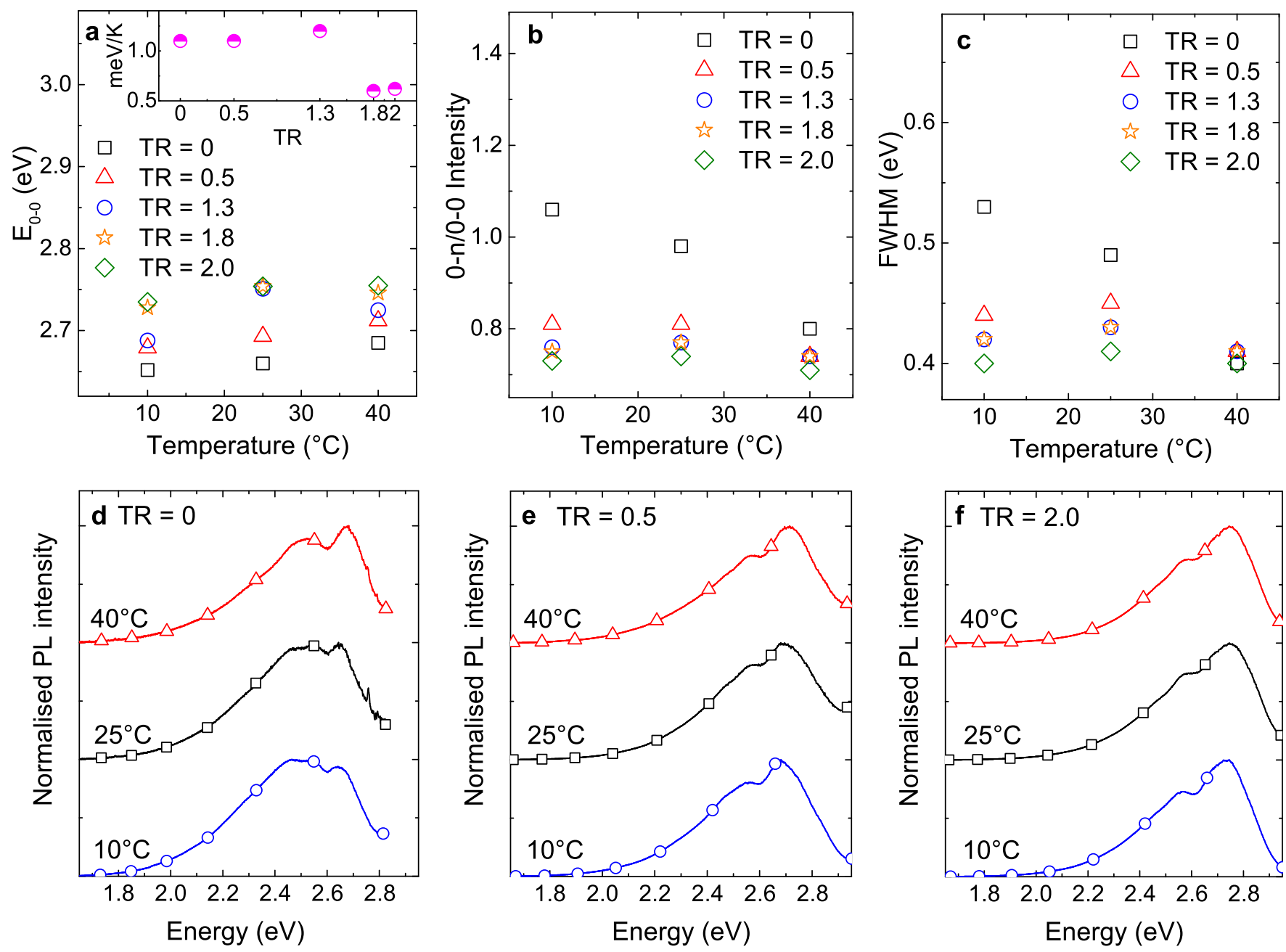

FIG. 2. Evolution of the parameters extracted from the PL spectra of PDV.Li and related polyrotaxanes with various threading ratios (TR $=0$ (black squares), 0.5 (red triangles), 1.3 (blue circles), 1.8 (orange stars), and 2.0 (green diamonds)) as a function of temperature: the energy of the $0-0$ transition (a) and the change of its position upon temperature variation (inset, pink half circles), $\mathrm{I}_{0-\mathrm{n}} / \mathrm{I}_{0-0}$ ratio, where 0 -n transition is that with the highest intensity excluding the 0-0 transition (b), and the full-width half-maximum (FWHM) of the emission (c). Stacked normalised PL spectra of $10^{-4} \mathrm{~g} 1^{-1}$ solution of PDV.Li (TR $=0$ ) (d) and PDV.Lic $\beta$-CD with different threading ratios, $\mathrm{TR}=0.5$ (e) and 2.0 (f), at $10^{\circ} \mathrm{C}$ (blue circles), $25^{\circ} \mathrm{C}$ (black squares), and $40^{\circ} \mathrm{C}$ (red triangles).

a higher degree of encapsulation of the polymers into cyclodextrins. In fact, at $40^{\circ} \mathrm{C}$ PDV.Li exhibits a relatively narrow spectrum $(0.40 \mathrm{eV})$ and a recovery of the $0-0$ intensity $\left(\mathrm{I}_{0-\mathrm{n}} / \mathrm{I}_{0-0}=0.80\right)$ quantitatively analogous to that of rotaxinated polymers with high threading ratio $(\mathrm{FWHM}=0.40 \mathrm{eV}$ and $\mathrm{I}_{0-\mathrm{n}} / \mathrm{I}_{0-0}=0.71$ ) as reported in Figs. 2(d) and 2(f), respectively.

Interestingly, Kasiouli and co-workers reported that solution of $10^{-4} \mathrm{~g} \mathrm{l}^{-1}$ of the PDV.Li and its rotaxinated counterpart with low TR (0.5) also show changes in the Raman spectra when the temperature is increased from $25^{\circ} \mathrm{C}$ to $40^{\circ} \mathrm{C}$ that are different with respect to the polyrotaxane with TR $>0.5 .^{20}$ They found a decrease in the relative intensity of the Raman band associated with the $\mathrm{C}-\mathrm{C}$ inter-ring stretch with respect to that of the ring $\mathrm{C}-\mathrm{H}$ interplane symmetric bend. Such a change in the Raman spectrum suggests a conformational rearrangement involving the dihedral angle between the adjacent phenyl rings and leading to a planarization of the polymer that would favour aggregation. This is contrary to our results reported in Fig. 2, clearly pointing to a reduction of interchain interactions at temperatures of $40^{\circ} \mathrm{C}$ or so. This trend is particularly evident for PDV.Li with $\mathrm{TR}=0$ and 0.5 . Incidentally, the slight increase in the
$0-0$ transition energy with temperature [Fig. 2(a)] also suggests reduction rather than increase of planarization (larger average torsional angles along the chains). We nevertheless expect that reduction or suppression of packing interactions in these systems far outweighs any torsional or vibrational "direct" effects on the chromophores photophysics.

Time-resolved spectroscopy is a more sensitive probe of aggregation than steady-state PL, and can unequivocally detect the presence or enhancement of interchain effects. We have therefore investigated the PL lifetime decays of PDV.Li and its rotaxinated counterparts recorded at $25^{\circ} \mathrm{C}$ to $40^{\circ} \mathrm{C}$ and then compared (see Fig. 3 and Table I). The lifetime of the unthreaded polymer and that of the polyrotaxane with TR $=0.5$ at $25^{\circ} \mathrm{C}$ are clearly multi-exponential (and fitted with 3 exponentials, see Table I) with a long-lived component that could be ascribed to interchain states. When the temperature is increased to $40^{\circ} \mathrm{C}$, the decays become biexponential with a fast component $(\sim 0.8 \mathrm{~ns})$ that could be attributed to intrachain species and a long-lived component ( 2.9 ns) typical of interchain species whose relative weight is reduced compared to that of the decay at $25^{\circ} \mathrm{C}^{7,22}$ Such long-lived component is still present in the polyrotaxane with TR $=1.3$ recorded at $25^{\circ} \mathrm{C}$. We note that the weight of 


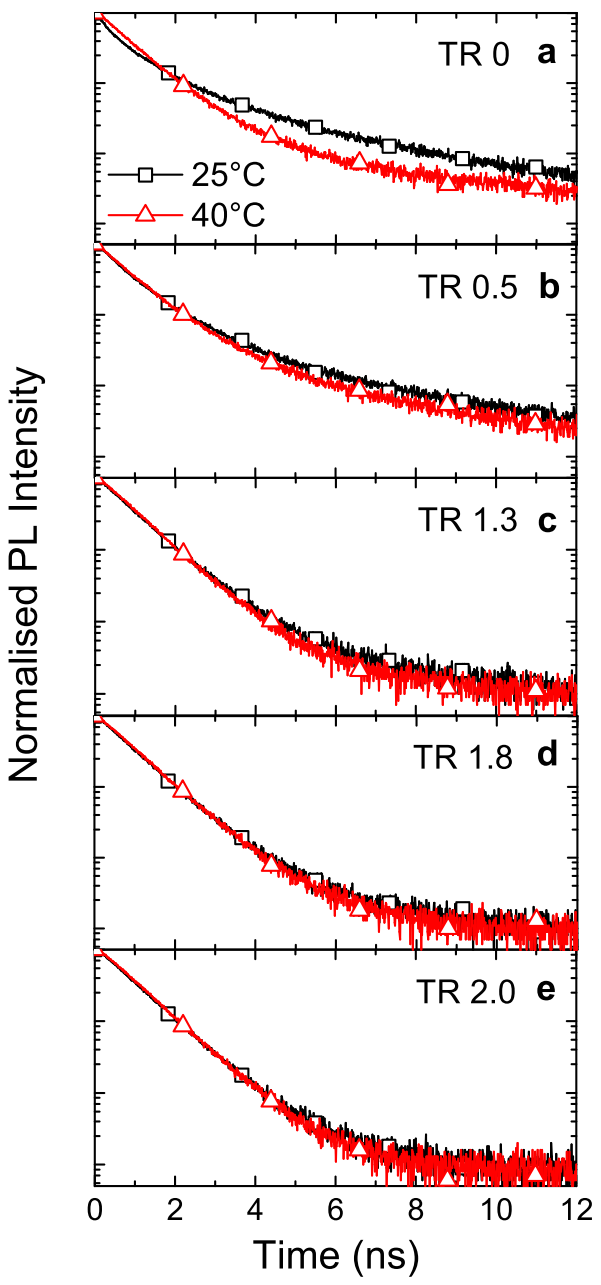

FIG. 3. PL lifetime taken at $2.53 \mathrm{eV}$ of $10^{-4} \mathrm{~g}^{-1}$ solution of PDV.Li $(\mathrm{TR}=0)$ (a) and PDV.Li $\subset \beta$-CD with different threading ratio, TR $=0.5$ (b), 1.3 (c), 1.8 (d), and 2.0 (e) at $25^{\circ} \mathrm{C}$ (black squares), and $40^{\circ} \mathrm{C}$ (red triangles).

the PL decay for the interchain species decreases as the temperature (and the threading ratio) increase, until it is not detectable any longer at $40^{\circ} \mathrm{C}$ for PDV.Li $\subset \beta$-CD with $\mathrm{TR}=1.3$. This indicates that interchain interactions that would lead to the formation of weakly emissive aggregates and/or weakly bound excimers are systematically disfavoured as the temperature increases. For rotaxanes with threading ratios higher than 1.3, we observe a mono-exponential decay

TABLE I. PL lifetime decay for PDV.Li and PDV.Li $\subset \beta$-CD aqueous solutions $\left(10^{-4} \mathrm{~g}^{-1}\right)$ at $25^{\circ} \mathrm{C}$ and $40^{\circ} \mathrm{C}$. The samples were excited at $3.34 \mathrm{eV}$, and the emission was collected at $2.53 \mathrm{eV}$.

\begin{tabular}{lccccc}
\hline \hline TR & Temperature $\left({ }^{\circ} \mathrm{C}\right)$ & $\tau_{1}(\mathrm{~ns})$ & $\tau_{2}(\mathrm{~ns})$ & $\tau_{3}(\mathrm{~ns})$ & $\mathrm{A}_{1}: \mathrm{A}_{2}: \mathrm{A}_{3}$ \\
\hline 0 & 25 & 2.95 & 0.87 & 0.26 & $18: 63: 19$ \\
& 40 & 2.91 & 0.82 & & $6: 94: 0$ \\
0.5 & 25 & 2.94 & 0.88 & 0.37 & $10: 71: 19$ \\
& 40 & 2.96 & 0.84 & & $7: 93: 0$ \\
1.3 & 25 & 2.91 & 0.85 & & $1: 99: 0$ \\
& 40 & & 0.85 & & \\
1.8 & 25 & & 0.85 & & \\
& 40 & & 0.85 & & \\
2.0 & 25 & & 0.85 & & \\
& 40 & & 0.85 & & \\
& & & & & \\
\hline \hline
\end{tabular}

both at $25^{\circ} \mathrm{C}$ and $40^{\circ} \mathrm{C}$ with a time constant of $\sim 0.85 \mathrm{~ns}$, typical of intrachain excitons. For such rotaxanes, interchain interactions are effectively minimised by the supramolecular encapsulation. We can therefore rule out that interchain species play a major role on the thermochromic behaviour reported in Fig. 2(a) for these "highly threaded" polyrotaxanes.

In this study, we have shown that the increase of the temperature from $10^{\circ} \mathrm{C}$ to $40^{\circ} \mathrm{C}$ affects the optical properties of PDV.Li and its polyrotaxanes in water solution. The 0-0 peak blue-shifts concomitantly to the decrease of the $\mathrm{I}_{0-\mathrm{n}} / \mathrm{I}_{0-0}$ ratio and the reduction of the FWHM of the PL emission as the temperature increases. This trend, that is particularly evident for the unthreaded polymer and the rotaxane with low $\mathrm{TR}(\mathrm{TR}=0.5$ and partly for $\mathrm{TR}=1.3)$, suggests a reduction of the interchain interactions between the polymer backbones. This is supported by time-resolved spectroscopy data for PDV.Li and its polyrotaxanes counterpart with $\mathrm{TR} \leq 1.3$ that clearly shows that the long-lived component in the PL decay, related to the interchain species, consistently diminishes as the temperature increases. For the most threaded rotaxanes ( $T R \geq 1.8$ ), the formation of interchain species are effectively minimized by cyclodextrin encapsulation.

This work was supported by the EC Seventh Framework Programme (FP7/2007-2013) under Grant Agreement No. 264694 (GENIUS). F.C. is a Royal Society Wolfson Research Merit Award holder.

${ }^{1}$ H. Jiang, P. Taranekar, J. R. Reynolds, and K. S. Schanze, Angew. Chem., Int. Ed. Engl. 48, 4300-4316 (2009).

${ }^{2}$ C. L. Zhu, L. B. Liu, Q. Yang, F. T. Lv, and S. Wang, Chem. Rev. 112, 4687-4735 (2012).

${ }^{3}$ S. W. Thomas, G. D. Joly, and T. M. Swager, Chem. Rev. 107, 1339-1386 (2007).

${ }^{4}$ B. J. Schwartz, Annu. Rev. Phys. Chem. 54, 141-172 (2003).

${ }^{5}$ M. Wykes, S. K. Park, S. Bhattacharyya, S. Varghese, J. E. Kwon, D. R. Whang, I. Cho, R. Wannemacher, L. Luer, S. Y. Park, and J. Gierschner, J. Phys. Chem. Lett. 6, 3682-3687 (2015).

${ }^{6}$ J. Gierschner and S. Y. Park, J. Mater. Chem. C 1, 5818-5832 (2013).

${ }^{7}$ S. Brovelli, G. Latini, M. J. Frampton, S. O. McDonnell, F. E. Oddy, O. Fenwick, H. L. Anderson, and F. Cacialli, Nano Lett. 8, 4546-4551 (2008).

${ }^{8}$ G. Latini, L. J. Parrott, S. Brovelli, M. J. Frampton, H. L. Anderson, and F. Cacialli, Adv. Funct. Mater. 18, 2419-2427 (2008).

${ }^{9}$ D. T. McQuade, J. Kim, and T. M. Swager, J. Am. Chem. Soc. 122, 5885-5886 (2000).

${ }^{10}$ U. Scherf and E. J. W. List, Adv. Mater. 14, 477-487 (2002).

${ }^{11}$ G. Wenz, B. H. Han, and A. Muller, Chem. Rev. 106, 782-817 (2006).

${ }^{12}$ A. Harada, A. Hashidzume, H. Yamaguchi, and Y. Takashima, Chem. Rev. 109, 5974-6023 (2009).

${ }^{13}$ G. Tregnago, M. Serri, S. Brovelli, S. O. McDonnell, P. Korniychuk, L. J. Wang, M. Wykes, D. Beljonne, A. Tracz, H. L. Anderson, and F. Cacialli, J. Mater. Chem. C 4, 10893-10902 (2016).

${ }^{14}$ C. Y. Tan, M. R. Pinto, M. E. Kose, I. Ghiviriga, and K. S. Schanze, Adv. Mater. 16, 1208-1212 (2004).

${ }^{15}$ P. Blondin, J. Bouchard, S. Beaupre, M. Belletete, G. Durocher, and M. Leclerc, Macromolecules 33, 5874-5879 (2000).

${ }^{16}$ J. M. Leger, A. L. Holt, and S. A. Carter, Appl. Phys. Lett. 88, 111901 (2006).

${ }^{17}$ C. Roux, J. Y. Bergeron, and M. Leclerc, Macromol. Chem. Phys. 194, 869-877 (1993).

${ }^{18}$ K. Yoshino, S. Nakajima, D. H. Park, and R. Sugimoto, Jpn. J. Appl. Phys., Part 2 27, L716-L718 (1988).

${ }^{19}$ W. R. Salaneck, O. Inganas, B. Themans, J. O. Nilsson, B. Sjogren, J. E. Osterholm, J. L. Bredas, and S. Svensson, J. Chem. Phys. 89, 4613-4619 (1988).

${ }^{20}$ S. Kasiouli, F. Di Stasio, S. O. McDonnell, C. P. Constantinides, H. L. Anderson, F. Cacialli, and S. C. Hayes, J. Phys. Chem. B 117, 5737-5747 (2013). 
${ }^{21}$ J. J. Michels, M. J. O'Connell, P. N. Taylor, J. S. Wilson, F. Cacialli, and H. L. Anderson, Chem. Eur. J. 9, 6167-6176 (2003).

${ }^{22}$ A. Petrozza, S. Brovelli, J. J. Michels, H. L. Anderson, R. H. Friend, C. Silva, and F. Cacialli, Adv. Mater. 20, 3218-3223 (2008).

${ }^{23}$ F. Cacialli, J. S. Wilson, J. J. Michels, C. Daniel, C. Silva, R. H. Friend, N. Severin, P. Samori, J. P. Rabe, M. J. O'Connell, P. N. Taylor, and H. L. Anderson, Nat. Mater. 1, 160-164 (2002).
${ }^{24}$ F. C. Spano, Acc. Chem. Res. 43, 429-439 (2010).

${ }^{25}$ G. Latini, A. Downes, O. Fenwick, A. Ambrosio, M. Allegrini, C. Daniel, C. Silva, P. G. Gucciardi, S. Patane, R. Daik, W. J. Feast, and F. Cacialli, Appl. Phys. Lett. 86, 011102 (2005).

${ }^{26}$ C. C. Wang, Y. Gao, A. P. Shreve, C. Zhong, L. Wang, K. Mudalige, H. L. Wang, and M. Cotlet, J. Phys. Chem. B 113, 16110-16117 (2009). 